\title{
EL ROL DE LAS UNIVERSIDADES PÚBLICAS FRENTE A LA REPONSABILIDAD SOCIAL UNIVERSITARIA
}

\author{
IVONNE MARÍA GIL OSORIO*
}

Recibido 10 de Julio de 2012 / Enviado para Modificación 15 de Septiembre de 2012 / Aceptado 2 de Noviembre de 2012

\section{RESUMEN}

El presente artículo tiene como propósito analizar el rol de las universidades públicas frente a la responsabilidad social universitaria, como un enfoque ético del vínculo mutuo entre universidad y sociedad, basado en planteamientos de autores como Vallares (1-2), Mejía (3); Sánchez (4), aportes de la Universidad construye país, y Asociación de universidades confiadas a la compañía de Jesús en América Latina (AUSJAL), entre otros. La metodología utilizada en la investigación fue un diseño de campo se enmarca estadísticamente como descriptiva, de campo no experimental. En la misma se estableció como unidad de análisis las dos universidades públicas del municipio de Riohacha (universidad de la Guajira y Universidad nacional abierta y a distancia), se estudió con una población finita de 34 sujetos informantes claves pertenecientes a los directivos de la universidad, como técnica e instrumento de recolección se utilizó la encuesta la cual fue sometida a la validez compuesta por 5 expertos, después de haber dado su veredicto, se procedió a la confiabilidad del instrumento mediante el coeficiente de coeficiente de Alpha de Cronbach ( $r t t=$ 0,90), considerado como de alta fiabilidad y los resultados de la investigación descritos por tablas de distribución frecuencia y porcentual obtenidos a través del programa Excel, que permitieron llegar a la conclusión que en estas entidades debe implementar una oficina de Responsabilidad social universitaria, con el propósito de contribuir al desarrollo interno y externo que los rodea

Palabras clave: Responsabilidad social universitaria, programas, vinculación y políticas

* Magister en Gerencia empresarial. Correo Electrónico: ivongil68@gmail.com 


\begin{abstract}
University Social Responsibility (RSU) is an ethical approach to the mutual bond between the university and society. This is an inalienable moral commitment that, at the same time generate new knowledge relevant to solving social problems, allows the direct application of scientific and technological knowledge as well as a more humane training, were used in this research contributions related theoretical theories of social responsibility among these authors can university mentioned Vallares (2007), Mejia (2009, Sanchez (2009), contributions from the University builds country, Association of Universities entrusted to the Jesuits in Latin America (AUSJAL). The research results will allow the public universities RSPU approach incorporating an institutional policy to guide change through its educational institutions, producing new models of sustainable development projects, teaching new alternatives that ensure that ensure the return of knowledge generated by applied research projects, and thus provide a contribution to efforts to rethink the link between university and society in Latin America.
\end{abstract}

Key words: Social Responsibility university programs, and policies linking

Classification Journal Economic Literature (JEL): I23, I21, M14.

\title{
INTRODUCIÓN
}

Las universidades tienen la responsabilidad de proporcionar formación científica, profesional, humanística, artística y técnica del más alto nivel, contribuir: a la competitividad económica para el desarrollo humano sostenible; promover la generación, la difusión del conocimiento en todas sus formas; contribuir a la preservación de la cultura nacional, desarrollando las actitudes y valores que requiere la formación de personas responsables, con conciencia ética, solidaria, reflexivas, innovadoras, críticas, capaces de mejorar la calidad de vida, consolidar el respeto al medio ambiente, a las instituciones del país y a la vigencia del orden democrático. En este sentido, es necesario resaltar que las actividades universitarias deben ser ejercidas con base a un conjunto de valores debidamente identificados y recogidos en su marco regulatorio, como son: la identidad, cultura nacional; el respeto al ser humano, su dignidad, su libertad; la libertad de discusión, el pluralismo ideológico, político, religioso; el espíritu democrático, la justicia social, la solidaridad humana; el rigor científico, la responsabilidad ética en la búsqueda para construcción del conocimiento; la creatividad, la criticidad, la integridad, la responsabilidad; la igualdad de oportunidades en el acceso a los beneficios de la educación superior. 
En vista de lo anterior, el presente artículo se considera de suma importancia para las universidades, ya que debe tratar de superar el enfoque de la extensión universitaria reflexionando sobre sus acciones en su entorno social, haciendo un análisis de su responsabilidad en los problemas crónicos de la sociedad. Para la consecución de este se acudió a la recolección de información a través de una encuesta, con el fin de poder vislumbrar la responsabilidad social en las universidades públicas.

\section{DESARROLLO}

La responsabilidad social empresarial es un conjunto de prácticas de la organización que forman parte de una estrategia corporativa y que, siguiendo fines racionales trata de evitar daños y/o producir beneficios para todas las partes interesadas en la actividad de la empresa (clientes, empleados, accionistas, comunidad, entorno entre otros) y que debe redundar en un beneficio de la organización como para la sociedad. Vallaeys (5).

Según la UNESCO (2007), en la Conferencia Mundial de educación superior, se plantea en unos de los puntos relacionados con la Responsabilidad Social Universitaria (artículo 6 de la declaración), la responsabilidad que tienen las universidades de tener orientaciones de largo plazo que permitan resolver las necesidades y aspiraciones sociales, inculcando esta responsabilidad a los estudiantes.

Igualmente señalan que la educación es la base de cualquier cambio que se pretenda en el estilo de pensamiento de las personas, que por tanto es la clave para transformar el modelo de desarrollo de cualquier sociedad. Considerando por tanto que el desarrollo social debe impulsar cambios a partir de la reflexión crítica sobre el modo de vida, implicando a los individuos en la toma de decisiones para trabajar de forma activa desde todos los grupos sociales en la construcción de un mundo cada vez mas razonable. Interacción de las Instituciones de Educación Superior con su entorno para la construcción de capital social y que este sea visto desde el frente político, institucional, social, económico y cultural.

Según Mejía (3), asume que unas de políticas de proyección social deben ir encaminadas a:

Aportar y difundir conocimiento sobre la problemática social, económica y política. 
Prestar servicios integrados para la solución de problemas comunitarias.

Realizar acciones cooperadas y servir de entidad para convocar, en regiones y espacios determinados a otras instituciones, sean estatales o privadas, con el objeto de aunar recursos y experiencias a la solución de problemas comunitarios.

Por otra parte Mejía (3), cita un aparte La Declaración Mundial de UNESCO sobre Educación Superior en el siglo XXI - visión y acción - nos invita a que esta debe ser más solidaria con el servicio a la comunidad, colaborar y contribuir a erradicar la pobreza, la intolerancia, la violencia, el analfabetismo, el hambre y sostenibilidad del medio ambiente.

\section{Programas de Responsabilidad Social Universitaria}

Una Universidad responsable debe: Tener una presencia en las realidades donde una Universidad responsable deba encargarse: presencia en lugares dentro y externos al Campus y enlazarse con agentes sociales significativos, que permeen y formen personas y también generen saberes con las realidades sociales. La Universidad es responsable si influye en la sociedad, canaliza la Influencia de una clara orientación transformadora de los ámbitos de vulnerabilidad social, económica y pública presente en nuestra sociedad.

Entonces extensión es presencia de las Universidades en las realidades que una Universidad debe encargarse y a través de procesos continuos de interacción e integración.

La Interacción debe medirse en términos de impacto como red, en la integración está basado en el enfoque de las capacidades institucionales (programas y proyectos específicos). Por otra parte el autor asume y dice que la universidades deben confluir hacia la extensión universitaria aplicando unos impactos que generan influencia en su entorno, los cuales se enuncian a continuación:

a) Impacto de la Universidad en la sociedad: Presencia a través de acciones significativas (valor transformador - redes) y sostenibles (identificar urgencias y retos de vulnerabilidad)

b) Impacto de la sociedad en la Universidad: Sólo pueden ser influidas universidades con capacidad de responder y que estén motivados para intervenir. 
Capacidad: Asumir retos sin bloqueos estructurales y así obtener resultados significativos.

Motivación: Intervenir a favor de terceros, conforme a su misión.

El concepto de Responsabilidad apunta a una reinstitucionalización de la sociedad, responder con vocación transformadora, proactiva y haciendo propuestas concretas de construcción de sociedad.

\section{Impactos de la Responsabilidad Social Universitaria}

\section{Impactos Organizacionales (Laboral y Ambiental):}

Excelente clima organizacional y relación sincrónica entre todos los actores al interior y exterior (código de buen gobierno corporativo, política labora justa, selección de proveedores con criterios de responsabilidad social y manejo sostenible del campus)

Campus responsable de sana convivencia (justicia, democracia y sostenibilidad).

\section{Impactos Educativos:}

Comprometimiento de todos los procesos de la Universidad (administración central, malla curricular y políticas de gestión del conocimiento) con la RS.

Formar a estudiantes como ciudadanos responsables y profesionales comprometidos con los más necesitados.

\section{Impactos Cognitivos:}

Se dirigen y lideran desde y hacia los docentes, quienes deben tener una actitud de facilitador y acometer un proceso de autoaprendizaje.

Gestiones sociales del conocimiento (producción y difusión), todas enseñan y aprenden a la vez.

\section{Impactos Sociales:}

Dirigidos a todos los actores del entorno: comunidad, sociedad civil, sector empresarial y sector público. La Universidad debe concertar la 
acción de los actores para producir impacto social y mejorar la calidad de vida de los ciudadanos.

Para Vallaeys (5), el concepto de responsabilidad social universitaria, como una gerencia ética e inteligente de los impactos que genera la institución basada en acciones concretas tomadas por las universidades en su entorno y contexto particular, de igual forma se describen a continuación los impacto que se producen con la acción u omisión en su gestión, en este sentido se distinguen 5 aéreas de impacto que las instituciones de educación superior promueven:

Impacto Organizacional, equivalente a la Responsabilidad Social Empresarial. Toda organización, por ser tal, produce impactos por medio de su gestión. Debería existir una ética de la Gestión, donde los valores estuvieran en el centro y donde esta ética se expresara en el clima laboral; en la relación con los clientes y proveedores; en la relación con los funcionarios -académicos y administrativos- $\mathrm{y}$ en la relación con sus estudiantes. En esta área de impactos hay que desarrollar un conjunto de políticas de responsabilidad social, relacionadas a cada uno de los aspectos antes señalados.

Impacto Ambiental. Las universidades producen impactos en el medio ambiente, tales como desechos o residuos contaminantes; uso indiscriminado de agua y energía; ruidos molestos; basuras sin separación en el origen. A su vez la universidad puede concienciar a su alumnado sobre los problemas ambientales a los que se enfrenta la sociedad y contribuir activamente a superarlos.

Impacto Educativo. Este ocurre en el procese de; cuando los alumnos se forman como ciudadanos democráticos; cuando la comunidad universitaria tiene la posibilidad de participar activamente en proyectos de servicio a la comunidad; cuando participan en la reflexión de las experiencias realizadas; cuando los miembros de la comunidad educativa se comprometen voluntariamente en proyectos de servicio; cuando hay un trabajo interdisciplinario en proyectos de servicio a la comunidad; cuando se producen mejoras continuas en los currículo a partir de las experiencias realizadas, etc.

Impacto Cognitivo. Cuando las líneas de investigación se abren a temas del desarrollo humano sostenible; cuando los proyectos de investigación asumen los temas étnicos; de género; de pobreza; de la discapacidad; cuando los proyectos de investigación incluyen la integración de diversas 
perspectivas disciplinarias; cuando hay un aprendizaje compartido con otros actores de la comunidad; cuando el conocimiento generado cumple con los estándares establecidos por la universidad sobre calidad y pertinencia; cuando los conocimientos generados son difundidos entre la comunidad académica, entre los actores relevantes en el tema y en la opinión pública en general, etc.

Impacto Social. Cuando la universidad abre sus puertas y recibe alumnos de los sectores más desfavorecidos de la población; cuando la universidad se hace cargo de sus alumnos reales y logra que se mantengan en el sistema con buenos rendimientos; cuando desarrolla programas para nivelar a los estudiantes en competencias básicas; cuando trabaja la deserción y la titulación oportuna; cuando cuenta con una serie de beneficios estudiantiles que permiten realizar lo descrito; cuando los proyectos sociales que emprende la universidad han cumplido con los objetivos que se plantearon; cuando la universidad mantiene convenios con actores sociales para desarrollar proyectos conjuntos; cuando las acciones emprendidas tienen un alcance significativo; cuando hay un presupuesto de la universidad destinado al vínculo con los actores sociales relevantes de la comunidad, etc.

De lo acuerdo a lo antes expuesto por los autores y la presente investigación, se relacionan ya que ambos señalan que las universidades deben cumplir con la responsabilidad de proporcionar formación científica, profesional, humanística, artística y técnica del más alto nivel. Con el fin de contribuir: a la competitividad económica y al desarrollo humano sostenible; promoviendo la generación, desarrollo y difusión del conocimiento en todas sus formas; para preservar la cultura, desarrollar actitudes y valores que se requieren para la formación de personas responsables, capaces de mejorar la calidad de vida, consolidar el respeto al medio ambiente, a las instituciones del municipio a través de la creación de redes sociales y las comunidades de aprendizaje y la cooperación mancomunada para conformar el tejido social que logrará la integración económica, social, política y cultural de la sociedad en general.

En este orden de ideas se presenta a continuación un esquema elaborado por Vallaeys F. (1) que puede ayudar a visualizar el carácter global y central del proyecto de responsabilidad social universitaria planteado en la" Declaración de Talloires". 


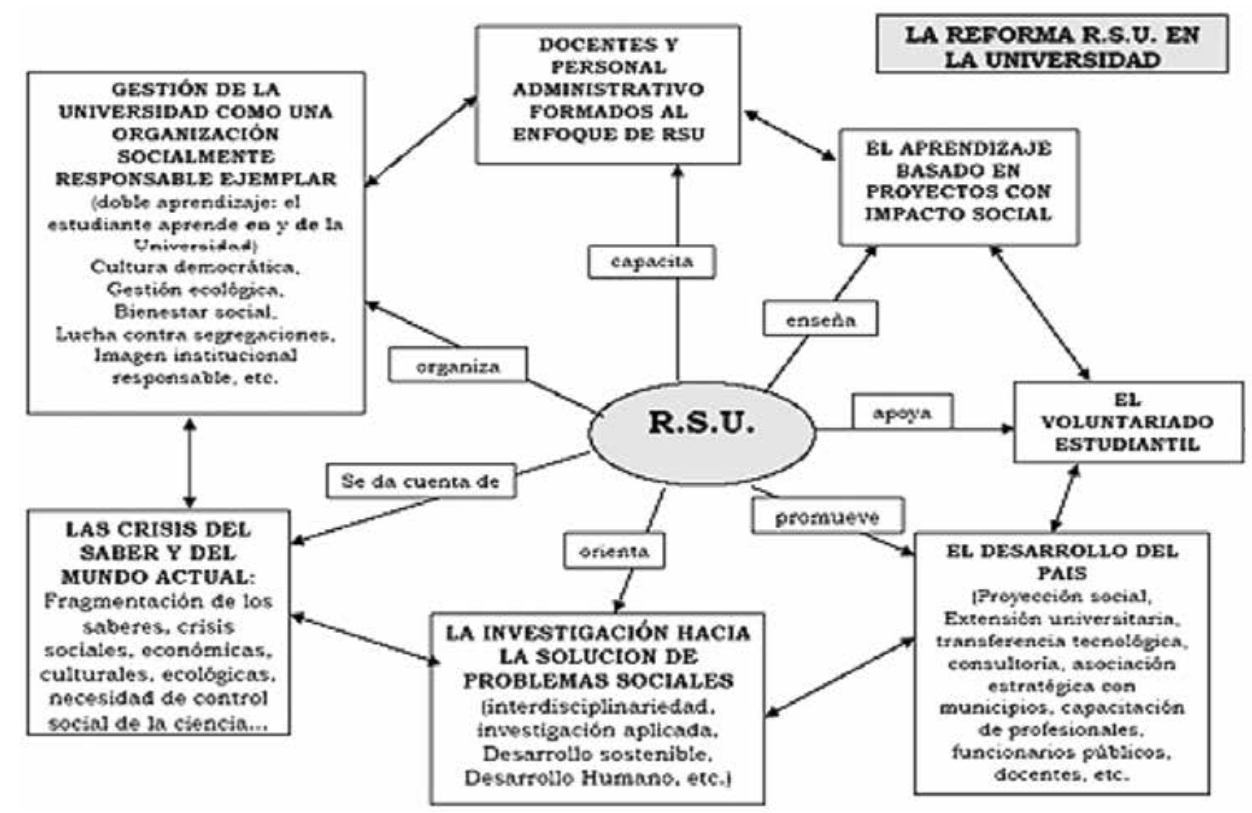

Fuente Vallaeys 2007

\section{Vinculación de los programas de Responsabilidad Social Universitaria}

Para Vallaeys F. (5), delinear estrategias generales de responsabilidad social universitaria es necesario establecer acciones institucionales tales como:

En lo que concierne a la Gestión interna de la Universidad: La meta es orientarla hacia la transformación de la Universidad en un pequeña comunidad ejemplar de democracia, equidad (supresión de las segregaciones y corrección de los privilegios), transparencia (política y económica), haciendo de ella, un modelo de desarrollo sostenible (política de protección del medio ambiente, uso de papel reciclado, tratamiento de los desechos).

En lo que concierne a la docencia : La meta es capacitar a los docentes en el enfoque de la Responsabilidad Social Universitaria, promover en las especialidades el Aprendizaje Basado en Proyectos de carácter social, abriendo el salón de clase hacia la comunidad social como fuente de enseñanza significativo, práctica aplicada a la solución de problemas reales. Donde el estudiante pueda aprender haciendo cosas socialmente útiles y formarse como ciudadano informado y responsable. Esto 
fomentará la creación de talleres de aprendizaje en las facultades, mayor articulación entre las disciplinas con mayor articulación entre la docencia, la investigación y la proyección social.

En lo que concierne a la investigación: La meta es promover la investigación para el desarrollo, bajo todas las formas posibles. Una estrategia posible es que la Universidad firme convenios con las gobernaciones, los municipios e invite a los departamentos de las diversas carreras a desarrollar investigaciones interdisciplinarias aplicadas con dichas localidades. Así, investigadores, docentes se encontrarían en el mismo lugar, trabajando sobre la misma problemática, desde sus especialidades respectivas, creando una sinergia de conocimientos, e interdisciplinariedad dejaría por fin de ser el "elefante blanco" del cual todos hablan pero que nunca nadie ha podido domar y montar.

En lo que concierne a la extensión: La meta es trabajar en interfaz con los departamentos de investigación y los docentes de las diversas facultades para implementar y administrar proyectos de desarrollo que puedan ser fuente de investigación aplicada y recursos didácticos para la comunidad universitaria. La idea es lograr una integración de la proyección social en el corazón de la institución, gracias a una Dirección Académica de Responsabilidad Social Universitaria que gestione las iniciativas estudiantiles y docentes, y pueda controlar su calidad. Tal unión estrecha entre proyección social, docencia e investigación promoverá, sin duda, el aumento significativo del voluntariado estudiantil, puesto que el alumnado habrá podido sacar provecho de un aprendizaje basado en proyectos sociales durante su formación.

Según lo anteriormente expuesto por Vallaeys F. (5), la articulación entre las líneas de acción institucional y las diversas carreras que propone cada universidad a través de los diferentes tipos de ejes temáticos que el campo del desarrollo ofrece y lo que las organizaciones no gubernamentales (ONGs) y las organizaciones internacionales se debe proponer una agenda social que incorpore desarrollo humano y calidad de vida, desarrollo económico, desarrollo tecno científico sostenible, desarrollo ciudadano y de la democracia, desarrollo de capacidades y cultura.

La Responsabilidad Social Universitaria, en el marco de las Universidades de AUSJAL (6), se ha de entender como la habilidad y efectividad de la universidad para responder a las necesidades de transformación de la sociedad donde está inmersa, mediante el ejercicio de sus funciones sustantivas: docencia, investigación y extensión. 
Al respecto señala la Universidad Construye País, Chile, el concepto de responsabilidad social universitaria, la capacidad que tiene la Universidad como institución de difundir y poner en práctica un conjunto de principios y valores, por medio de cuatro procesos claves: gestión, docencia, investigación y extensión.

Para Vallaeys (5), AUSJAL (6) y la Universidad Construye País (7), estas uniones deben estar animadas por la búsqueda de la promoción de la justicia, la solidaridad y la equidad social, mediante la construcción de respuestas exitosas para atender los retos que implica promover el desarrollo. Por lo tanto, la Responsabilidad Social Universitaria debe ser un eje transversal del quehacer de las universidades. Las autoridades universitarias deben garantizar la coherencia en la formulación e implementación de las acciones de Responsabilidad Social las tres funciones sustantivas señaladas a fin de que están sean efectivas.

La Responsabilidad Social Universitaria, en el marco de las Universidades de AUSJAL (6), se ha de entender como la habilidad y efectividad de la universidad para responder a las necesidades de transformación de la sociedad donde está inmersa, mediante el ejercicio de sus funciones sustantivas: docencia, investigación y extensión

Estas funciones deben estar animadas por la búsqueda de la promoción de la justicia, la solidaridad y la equidad social, mediante la construcción de respuestas exitosas para atender los retos que implica promover el desarrollo. Por lo tanto, la Responsabilidad Social Universitaria debe ser un eje transversal del quehacer de las universidades de AUSJAL. Las autoridades universitarias deben garantizar la coherencia en la formulación e implementación de las acciones de Responsabilidad Social las tres funciones sustantivas señaladas a fin de que están sean efectivas. En cuanto a los criterios propios de las universidades se propone considerarlos a la luz de sus tres funciones sustantivas:

Docencia: Es el espacio donde el estudiante a la vez que aprende particularidades disciplinares se entrena en su capacidad argumentativa, se forma en criterio y adquiere nuevos elementos para observar, relacionarse e incidir en el mundo social. La actividad formativa debe incorporar los espacios para la experiencia vivencial.

Investigación: La investigación en nuestras universidades debe favorecer la construcción de redes de conocimiento entre las diferentes disciplinas e instituciones. Las agendas de investigación deberán incorporar, entre 
sus líneas de trabajo, el análisis de temas que conduzcan al desarrollo de propuestas concretas como respuesta a problemáticas de la realidad.

Extensión: Es sacar el conocimiento de los claustros y ponerlo al servicio de quienes no están dentro de él, de diversas maneras. Es la oportunidad de realizar el quehacer educativo extramural, en estrecha relación con las necesidades sociales. Esta es la función que permite a las instituciones educativas no aislarse del mundo del cual hace parte, a la vez que aportar en concreto a su transformación. Además, es la oportunidad de poder evidenciar las transformaciones que se pueden hacer en interacción con la sociedad desde la academia. La extensión es una excelente oportunidad para propiciar un mayor diálogo de saberes con otros actores sociales que al igual que las universidades, están haciendo esfuerzos para construir sociedades más viables.

Se asume por lo anterior que en este sentido, una perspectiva de responsabilidad social en la educación superior favorece la función de la universidad, en la medida en que la proyecta y la pone en contacto con la realidad: le da oportunidad de probar, en situaciones concretas, el grado de eficiencia profesional de sus egresados o futuros profesionales y le permite, sobre la base de estas experiencias de servicio a la comunidad, actualizar su currículum y sus técnicas según las exigencias de la realidad.

Por lo tanto, se requiere formar en los alumnos la capacidad de comprometerse. En este sentido, debe fomentarse la iniciativa y la responsabilidad por la propia vida, es decir, la proactividad. Es preciso, además, desarrollar un modo de pensamiento capaz de escucha y dialogo, de tomar distancia ante los problemas situaciones, así como de ver a través de los ojos del otro, un pensamiento crítico, holístico y sistémico, capaz de identificar las partes de un todo y su interdependencia; un pensamiento dialéctico, que reconozca y trabaje efectivamente con contradicciones, y una sabiduría práctica, para poder reconocer el conjunto sin perder los detalles.

\section{POLITICAS DE RESPONSABILIDAD SOCIAL}

Vallaeys, (5) afirma que la Responsabilidad Social Universitaria (RSU) como: "Una política de calidad ética del desempeño de la comunidad universitaria (estudiantes, docentes y personal administrativo) a través de la gestión responsable de los impactos: educativos, cognitivos, laborales, ambientales que la universidad genera, en un dialogo participativo, con la sociedad para promover el desarrollo humano. 
Por otra parte Vallaey, (2), que a través de la gestión responsable de los impactos específicos de las universidades estás conducen a la definición de unas políticas articuladas para la promoción de:

1. Calidad de vida institucional ejemplar (laboral y medioambiental), al promovercomportamientoséticos, democráticosymedioambientalmente adecuados para tener un Campus responsable y congruente con los valores declarados de la Universidad.

2. Formación académica integral de ciudadanos responsables y capaces de participar del desarrollo humano sostenible de su sociedad (mediante la enseñanza de los conocimientos esenciales a la vida ciudadana responsable en el siglo XXI, uso de métodos de aprendizaje relacionados con proyectos sociales, Aprendizaje-Servicio, etc.).

3. Gestión social del conocimiento capaz de superar la inaccesibilidad social del conocimiento, y la irresponsabilidad social de la ciencia, en un mundo en el cual la calidad de vida depende cada vez más del acceso al conocimiento pertinente (selección y producción de conocimientos socialmente útiles y difundidos adecuadamente hacia la comunidad, con métodos de investigación participativos y democráticamente elaborados).

4. Participación social solidaria y eficiente (creación de conocimientos y procesos participativos con comunidades para la solución de problemas urgentes de la agenda social del Desarrollo, proyectos sociales y medioambientales, Comunidades de Aprendizaje mutuo para el Desarrollo, creación de nuevas redes de Capital Social para la toma de decisión ilustrada a favor del Desarrollo Humano Sostenible, etc.).

Es obvio que estas cuatro políticas se articulan entre sí, pueden juntas potenciarse mutuamente y orientar eficazmente la Universidad hacia su responsabilidad social.

Por su parte AUSJAL (6), organiza las políticas de responsabilidad social en tres ejes fundamentales:

La Formación de estudiantes:

Orientar los programas y los planes de estudio al contexto o realidad que los circunscribe, con el propósito de sensibilizar a nuestros estudiantes respecto a su entorno social. De esta forma, pretendemos desarrollar una conciencia crítica y claridad en el por qué y el para qué del saber.

Promover, incentivar y motivar la capacidad de respuesta de nuestros estudiantes frente a los problemas sociales, políticos, económicos y 
culturales de su entorno, comprometiéndose de manera creativa y constructiva desde espacios institucionales, creados para tal efecto. Se trataría de desarrollar el liderazgo, entendido como la capacidad de construir futuro para el bienestar general.

Asegurar la formación ética de nuestros futuros profesionales capacitándolos en función de una mejor comprensión de la realidad social. Se trata de crearles la capacidad de alerta frente a las implicaciones éticas de su actuación personal y profesional.

Garantizar en los planes de estudio, cursos obligatorios y electivos, cuyos contenidos y propuestas pedagógicas se encuentren efectivamente articulados con los problemas claves que se plantean a la Responsabilidad Social Universitaria.

Ofrecer a los estudiantes oportunidades vivenciales de servicio en las prácticas profesionales y sociales.

Sobre la actividad académica,

Desarrollar espacios interdisciplinarios para analizar y comprender los problemas que aquejan a nuestra realidad local y regional.

Definir una agenda temática de investigación orientada a atender los problemas sociopolíticos y económicos cuyos impactos afectan la productividad y la convivencia social. Estos temas pueden ser, entre otros:

i) Institucionalidad, ciudadanía, democracia y participación; ii) sociabilidad y solidaridad para la convivencia; iii) bien común y equidad social; iv) desarrollo sostenible y medio ambiente; v) aceptación y aprecio a la diversidad; vi) educación y salud.

Desarrollar permanentemente pensamiento, debate y análisis sobre el acontecer nacional y regional a través de la creación de instancias y espacios de interdependencia y diálogo.

Esto con criterios de pertinencia y en concordancia con la interpretación de las demandas que plantea el contexto sociopolítico y cultural.

Sobre la gestión universitaria:

Desarrollar espacios e instancias donde se difunda - tanto en forma eclesial como en forma académica - los principios éticos del humanismo cristiano. 
Desarrollar mecanismos de inducción y desarrollo de los recursos humanos (personal académico, administrativo y directivo) consistentes con los fines institucionales y la Identidad, en orden a su formación ética y a su sensibilización frente a la Responsabilidad Social Universitaria.

Diseñar y ejecutar estrategias comunicacionales, internas y externas, que faciliten el posicionamiento de la Responsabilidad Social Universitaria.

La gestión social de la universidad es un componente de su misión derivado de los otros dos, la formación y el conocimiento. La universidad debe ser sensible a las urgencias que le plantea el entorno, pero desde esas urgencias no se puede definir su misión; es decir, la economía y el estado deben hacer demandas a la universidad, pero no puede aceptarse que le den una orientación global

La respuesta de la universidad al entorno regional se inscribe en la cuestión más amplia de la proyección de la educación superior en el ambiente social. El punto de vista que aquí se plantea va en la dirección de propender por una activa participación de la universidad en la vida de la comunidad, pero conservando siempre la perspectiva de lo que propiamente constituyen su naturaleza y misión. El ejercicio de la buena academia no dista sino que compromete con los asuntos que interesan al medio externo.

Las universidades públicas, por lo general no tienen gran participación con el entorno, trayendo como consecuencia la poca relevancia en cuanto a los problemas que tienen la comunidad, lo cual afecta al desarrollo socioeconómico de la región; considerando que el principio de las universidades deben ser fieles a las tareas que le competen por la cual fueron creadas que es impartir educación superior con el propósito de articular cuatro funciones fundamentales en esta materia que son: la gestión, docencia, investigación y extensión.

No obstante ocurre con mucha frecuencia que estas funciones, esenciales en la vida universitaria, exista una desarticulación entre ellas, originando consecuentemente la falta de compromiso de los órganos internos de ellas, y a su vez causando un efecto negativo tanto interno como externo.

Todo lo anterior significa que una articulación clara de la docencia e investigación la que lleva impactar a la sociedad y este es el camino que produce extensión, siendo esta integración la que promueve un impacto en el medio. La extensión universitaria como su nombre lo indica es una prolongación del quehacer universitario siendo esta un apéndice de las instituciones de educación superior. 


\section{CONCLUSIONES}

La culminación de esta investigación permitió profundizar y reflexionar acerca de la participación de la comunidad educativa universitaria en el logro de metas, lo cual debe darse en el marco de un ejercicio responsable del juicio de los actos y del respeto a la libertad, la ética y la tolerancia dentro de una perspectiva de compromiso y pertinencia institucional.

Se concluye que las instituciones de educación superior públicas, no están cumpliendo con su misión, porque Responsabilidad Social Universitaria (RSU) es un enfoque ético del vínculo mutuo entre universidad y sociedad. Se trata de un compromiso moral irrenunciable que, a la par que genera nuevo conocimiento relevante para la solución de los problemas sociales, permite la aplicación directa del saber científico y tecnológico, así como una formación profesional más humanitaria.

La educación superior siempre se ha considerado como un factor de movilidad social, entendida ésta como la posibilidad que tendrá el egresado universitario de ascender en la escala social por el hecho de estar mejor dotado para enfrentar el mundo del empleo. Sin embargo, Ante una sociedad desigual y en la que el acceso a la enseñanza universitaria es más bien un privilegio, la universidad no puede abstraerse de dicha realidad y permitir que sus egresados abandonen las aulas con la sensación de que obtuvieron algo para sí mismos únicamente. Resulta central que entiendan que han recibido mucho de la sociedad y que tienen la responsabilidad de transformar esa realidad.

Las acciones de la Responsabilidad social de las Universidades, deben estar orientadas a lograr ciudadanos probos y honestos que concluyan su carrera profesional como conocimientos que validen las incumbencias para los cuales sus títulos lo habilitarán personas comprometidas con la sociedad de la que forma parte y a la cual volcarán sus conocimientos para mejorar su calidad de vida, tratando de solucionar los problemas que esa sociedad afronta.

\section{REFERENCIAS BIBLIOGRÁFICAS}

1. Vallaeys, Francoys (2007), Hacia la construcción de indicadores de Responsabilidad social universitaria. PUCP

2. Vallaeys, Francoys (2007). Programas de apoyo de iniciativas de responsabilidad social universitaria, Ética de y desarrollo. Banco Interamericano de Desarrollo. 
3. Mejía Pardo F (2009), Como Entendemos la Responsabilidad Social Universitaria. Director de Extensión, Universidad del Rosario, Bogota D,C. Colombia, www.urosario.edu.co/extension.

4. Sánchez González C. (2009) Responsabilidad Social Universitaria (rsu) en el contexto del cambio de la educación superior" Universidad Nacional Autónoma de México.

5. Vallaeys, Francoys (2007). Responsabilidad social universitaria, propuesta para una definición madura y eficiente. Tecnológico de Monterey, México

6. Asociación de universidades confiadas a la compañía de Jesús en América Latina AUSJAL (2005), políticas e indicadores de Responsabilidad social Universitaria.

7. Universidad construye país (2006). Responsabilidad social Universitaria. Una manera de ser universidad. Teoría y práctica en la experiencia chilena. Ed Gráfica funny, Santiago de Chile.

\section{BIBLIOGRAFÍA CONSULTADA}

Carrillo Hernández, Joaquín, (2011), El rol de las universidades públicas frente a la responsabilidad social universitaria, Magister Scientiarum en Gerencial de Proyectos de Investigación y Desarrollo. La Guajira Colombia.

Debate temático. (2006) auspiciado por la UNESCO sobre"responsabilidad Social, autonomía y libertad académica de las universidades en el Marco de la "Conferencia Mundial sobre Educación superior".

Gil Osorio, Ivonne Maria (2011), El rol de las universidades públicas frente a la responsabilidad social universitaria. Economista, Magister Scientiarum en Gerencial Empresarial. La Guajira Colombia

Proyecto "Universidad Construye País" (2006) "Responsabilidad Social Universitaria. Aproximación al concepto y su práctica" Santiago de Chile, Chile. 